Enrique C. Papa II, MD Emmanuel S. Samson, MD

Francisco A. Victoria, MD

Department of Otolaryngology-Head and Neck Surgery Ospital ng Maynila Medical Center
Correspondence: Dr. Enrique C. Papa II

Department of Otolaryngology-Head and Neck Surgery

Ospital ng Maynila Medical Center

Quirino Ave. cor Roxas Blvd., Malate, Manila 1004

Philippines

Phone: (632) 5246061 local 220

Email: enriq9tales@yahoo.com

Reprints will not be available from the author.

The authors declared that this represents original material that is not being considered for publication or has not been published or accepted for publication elsewhere in full or in part, in print or electronic media; that the manuscript has been read and approved by all the authors, that the requirements for authorship have been met by each author, and that each author believes that the manuscript represents honest work.

Disclosures: The authors signed disclosures that there are no financial or other (including personal) relationships, intellectual passion, political or religious beliefs, and institutional affiliations that might lead to a conflict of interest.

Presented at the Descriptive Research Contest Philippine Society of Otolaryngology Head and Neck Surgery, Natrapharm, The Patriot Building September 14, 2011

\section{Glottal Function Index and GRBAS Scale of Patients Undergoing Vocal Cord Medialization: A Series of Five Patients}

\begin{abstract}
Objective: Vocal cord paralysis or immobility is a debilitating condition that may result from neural injury or mechanical fixation of the vocal cord (VC). When permanent, therapy is aimed at improving closure by modifying the position of the vocal cord. Whatever surgical intervention is chosen, pre - and post - operative voice evaluation is important. This study aimed to investigate the usefulness of the Glottal Function Index (GFI) and Grade, Roughness, Breathiness, Asthenia, Strain (GRBAS) Scale in the evaluation of treatment outcomes in patients with unilateral vocal cord paralysis (UVCP) who underwent medialization thyroplasty type 1 with a modified lock-in soft silicone implant.
\end{abstract}

\section{Methods:}

\section{Design: Descriptive Case Series \\ Setting: Tertiary Government Hospital \\ Patient: Five}

Results: Five patients ( 3 females, 2 males) consulted due to hoarseness underwent rigid endoscopy. Four (2 right, 2 left) had unilateral paramedian VC paralysis while one had bilateral paresis with bowing of the left vocal cord. One of those with left VC paralysis was diagnosed as idiopathic; the four were iatrogenic ( 3 from thyroid surgery, 1 from multiple surgical procedures). All patients underwent medialization thyroplasty type 1 using locked-in soft silicone implant. The GFI and GRBAS scale were utilized for pre-operative and post-operative perceptual evaluation of voice. The GFI showed severe glottic insufficiency among all five patients prior to surgery with improvement of subjective symptoms one day and one week post-surgery in four patients. Likewise, the Hirano GRBAS scale showed improvement of voice quality and correlated well with the improvement of the patient's subjective symptoms from the GFI scores. However, case 5 with bilateral vocal cord paresis, showed no improvement of voice quality despite recovery from subjective symptoms.

Conclusion: For glottal insufficiency, perceptual voice evaluation using self-administered GFI and GRBAS scale assessment are important parameters in determining quality of life among patients with glottal insufficiency undergoing medialization laryngoplasty.

Keywords: hoarseness, unilateral vocal cord paralysis, medialization thyroplasty, Glottal Function Index, Hirano GRBAS Score 
Vocal cord paralysis or immobility is a debilitating condition that causes great impact on an individual. It may result from neural injury or mechanical fixation of the vocal cord (VC). The most common causes are previous surgery to the head, neck or chest, and neoplasms of the head, neck or thorax while a minority of causes include trauma, central nervous system diseases, inflammatory diseases or idiopathic origins. ${ }^{1,2}$ When vocal cord paralysis is permanent, therapy is aimed at improving closure by modifying the position of the vocal fold. Whatever surgical intervention is chosen (injection thyroplasty, medialization thyroplasty, arytenoid adduction or laryngeal reinnervation), preoperative and postoperative voice evaluation is important. This study aims to investigate the usefulness of the Glottal Function Index (GFI) and Grade, Roughness, Breathiness, Asthenia, Strain (GRBAS) Scale in the evaluation of treatment outcomes in patients with unilateral vocal cord paralysis (UVCP) who underwent medialization thyroplasty type 1 with a modified lock-in soft silicone implant.

\section{MATERIALS AND METHODS}

Study Design: Descriptive Case Series

Setting: Tertiary Government Hospital

Patients: Five patients

\section{Patient Selection}

With institutional review board approval, all patients diagnosed with unilateral vocal cord paralysis between January 1, 2008 and December 31,2010 from whom informed consent was obtained were included in the study with no further inclusion or exclusion criteria.

\section{Methods of voice examination}

The Glottal Function Index (GFI) a validated 4-item self-administered survey was used to evaluate glottal insufficiency using the cut-off for an abnormal GFI at 4 (mean +2SD). ${ }^{2}$ (Table 1)

Table 1. The Glottal Function Index

\begin{tabular}{|c|c|c|c|c|c|c|}
\hline \multicolumn{7}{|c|}{ A score $>4$ may indicate a significant voice disorder } \\
\hline \multicolumn{7}{|c|}{ Within the last month, how did the $0=$ no problem } \\
\hline following problems affect you? & \multicolumn{6}{|c|}{$5=$ severe problem } \\
\hline 1. Speaking took extra effort & 0 & 1 & 2 & 3 & 4 & 5 \\
\hline $\begin{array}{l}\text { 2. Throat discomfort of pain after using your } \\
\text { voice }\end{array}$ & 0 & 1 & 2 & 3 & 4 & 5 \\
\hline 3. Vocal fatigue & 0 & 1 & 2 & 3 & 4 & 5 \\
\hline 4. Voice cracked or & 0 & 1 & 2 & 3 & 4 & 5 \\
\hline
\end{tabular}

Reproduced with permission from Back KK, Befalsky PC, Wayslil K, Postma GN, Koufman JA Validity and reliability of the Glottal Function Index. Arch Otolaryngol Head Neck Surg, 2005 Nov; 131(11):961-4.
The Hirano GRBAS scale ${ }^{3}$ for perceptual analysis of voice was also used. In a quiet audiometry room, the patients were instructed to read at a comfortable loudness level at a comfortable rate. Recordings were performed using a Sony Handicam DCR-SR45 (Sony Corp., USA). Preoperative and post-operative voice recordings of the patients were taken one day before, one day and one week after surgery and graded by a single observer (the surgeon). Voice was scored using the parameters of the GRBAS system: Grade $=$ overall degree of deviance of voice, Roughness= irregular fluctuation of the fundamental frequency, Breathiness $=$ turbulent noise produced by air leakage, Aesthenia= overall weakness of the voice, and Strain $=$ impression of tenseness or excess effort. Each parameter was scored on a scale of 0 to 3 ( 0 was considered normal, 1 with slight disturbance, 2 with moderate disturbance, and 3 with severe disturbance). ${ }^{6}$

\section{Surgical Technique}

Medialization Thyroplasty was done in all five cases by a single surgeon, employing the surgical technique of Ishiki. $\mathrm{A}$ horizontal incision was made a few millimeters from the midline anterior neck area approximating the location of the middle of the thyroid cartilage. Flaps were developed and carried down to expose the entire height of the thyroid cartilage. The first case utilized a $9 \times 4 \mathrm{~mm}$ window below an imaginary line midway between the superior and inferior border of the right thyroid ala while the latter four cases had a smaller window width of $4 \times 4 \mathrm{~mm}$. The dimensions of the soft-silicone implant were modified to fit the subperichondrial window. Intraoperative voice assessment was done while adjusting the implant. Excess silicon was then shaved. The operative site was closed in layers using chromic 3-0 and the skin approximated using silk $4-0$.

\section{RESULTS}

From 2008-2010, five patients (2 males, 3 females, aged 34-56, mean 40) consulting for hoarseness were diagnosed with unilateral vocal cord paralysis by rigid endoscopy. (Table 2) Four (2 right, 2 left) had unilateral paramedian VC paralysis while one had bilateral paresis with bowing of the left vocal cord. One of those with left VC paralysis was diagnosed as idiopathic; the four were iatrogenic (3 from thyroid surgery, 1 from multiple surgical procedures). The duration of paralysis ranged from 8 months to 18 years, all presented with hoarseness, with one each experiencing aspiration and stridor as well. (Table 2) All patients underwent medialization thyroplasty type 1 using locked-in soft silicone implant.

The self-administered Glottal Function Index (GFI) and observeradministered Hirano GRBAS scale were utilized for pre-operative 
Table 2. Patient Profiles

\begin{tabular}{|c|c|c|c|c|c|c|c|}
\hline & Age & Sex & Symptoms & Duration & VC Laterality & VC Position & Etiology \\
\hline Case 1 & 50 & Female & Hoarseness & 3 years & Right & Paramedian & latrogenic \\
\hline Case 2 & 56 & Male & $\begin{array}{c}\text { Hoarseness and } \\
\text { aspiration to liquids }\end{array}$ & 8 months & Left & Paramedian & latrogenic \\
\hline Case 3 & 42 & Male & Hoarseness & 10 months & Left & Paramedian & Idiopathic \\
\hline Case 4 & 37 & Female & Hoarseness & 2 years & Right & Paramedian & latrogenic \\
\hline Case 5 & 34 & Female & $\begin{array}{l}\text { Hoarseness and } \\
\text { stridor } \\
\text { (s/p Keel insertion) }\end{array}$ & 18 years & Left & $\begin{array}{l}\text { Bilateral paresis with } \\
\text { bowing of left VC }\end{array}$ & latrogenic \\
\hline
\end{tabular}

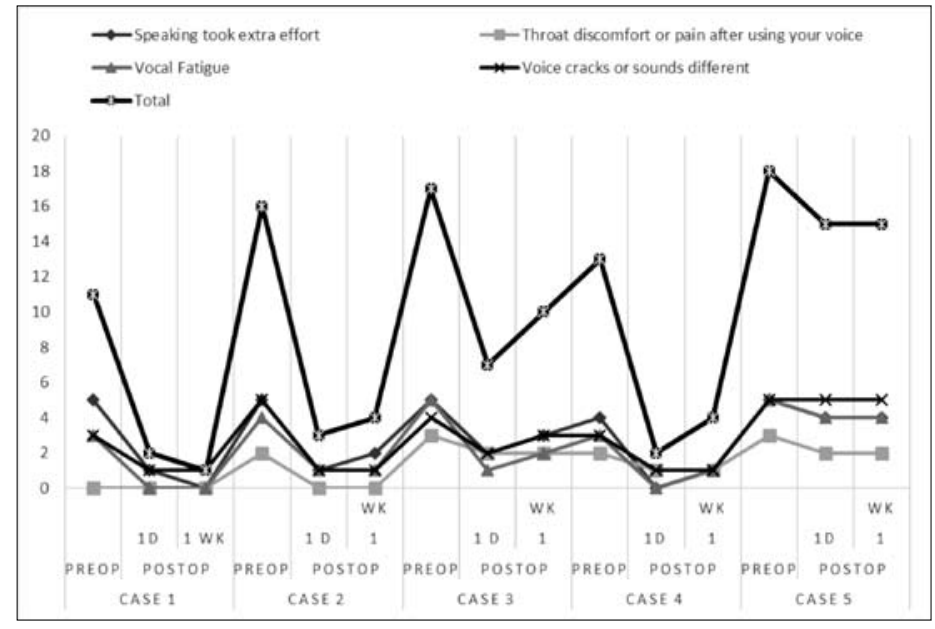

Figure 1. Glottal Function Index showing severe glottic insufficiency among all five patients prior to surgery with improvement of subjective symptoms one day and one week post-surgery.

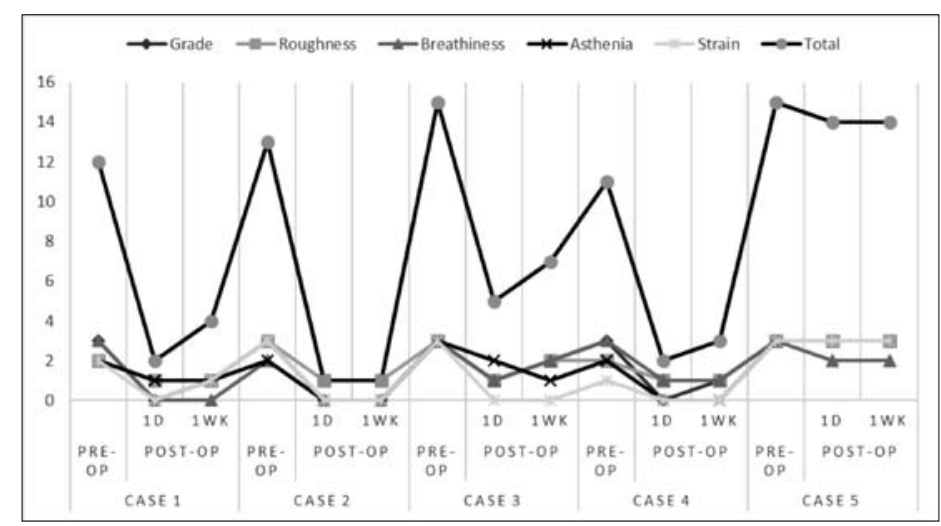

Figure 2. Hirano GRBAS Scale showing improvement of voice quality that correlated well with the improvement of the patient's subjective symptoms from the GFI scores, except in the case of patient number 5 where no improvement of voice was noted despite minimal improvement in subjective symptoms. and post-operative perceptual evaluation of voice. The GFI showed severe glottic insufficiency among all five patients prior to surgery with improvement of subjective symptoms one day and one week post-surgery in four patients. (Figure 1) Likewise, the Hirano GRBAS scale showed improvement of voice quality and correlated well with the improvement of the patient's subjective symptoms from the GFI scores,except in the case of patient number 5 where no improvement of voice was noted despite minimal improvement in subjective symptoms. (Figure 2)

Only one patient had wound infection 1 week post-operatively and following oral antibiotics, granulation tissue that developed at the operative site was treated with excision and primary closure.

\section{DISCUSSION}

Glottic insufficiency is one of the common contributing factors in patients complaining of dysphonia. This condition may be brought about by unilateral vocal cord paralysis or paresis, presbylaryngis and other causes. Glottic insufficiency yields a major impact on quality of life with the potential for significant morbidity and mortality. ${ }^{2}$

Our patient demographics although small reflected the common causes of vocal cord paralysis with iatrogenic causes being most common with thyroid surgery accounting for the majority of UVCP. However, other non-thyroid surgical procedures when combined still far outnumber thyroid surgery related vocal cord paralysis. Idiopathic vocal cord paralysis affects the left vocal cord more than the right due to anatomic reasons, and this holds true in our patients. ${ }^{1}$

The use of various surveys for assessment of voice rehabilitation outcome provides an objective insight to a patient's initial disability and perceived benefit following surgery. The Voice Handicap Index (VHI) developed by Jacobson ${ }^{5}$ delivers a multifaceted assessment as it gives information on the functional, emotional and physical attributes 
of a patient with voice disorder although it has been observed that "this 30-item self-administered test often deters patients from finishing the task and requires a relative amount of patience and comprehension."2 The Glottal Function Index (GFI) was alternately used as it is a reliable, reproducible, 4-item, self-administered symptom index used effectively to evaluate patients and "its results are comparable to those of the VHI and provide a significant advantage for being brief, symptom focused and easily completed." It also provides quick and easy administration and correlates well as an adjunct instrument with other perceptual tests. Our study found that reduction in symptoms as related by the patients gave positive reinforcement on treatment success whether or not significant improvement of voice was achieved.

The Hirano GRBAS scale which is examiner-based is the gold standard in perceptual analysis of voice. ${ }^{5}$ It has a significant correlation with the voice parameters quantified by the Multi-Dimensional Voice Program (MDVP). ${ }^{4}$ The results we obtained using GRBAS strengthened our findings with GFI. Both tests showed improvement of symptoms and voice quality post-operatively. However, it must be acknowledged that the lack of an independent blinded observer other than the surgeon is a major limitation of this study and blinded evaluation by a speech pathologist is recommended for future studies.

Another limitation is the early post-operative evaluation at one day and one week respectively. A change in voice quality one week postoperatively compared to one day post-operatively may be explained by formation of granulation tissue or decreased edema of laryngeal tissue which causes an increased glottic gap. ${ }^{6}$ Even though postoperative recovery may occur rapidly from the first week to three months, optimal voice quality may not be obtained for at least three months following surgery since further reduction in laryngeal edema or hematoma may increase the glottic gap and may need further adjustment of the implant. ${ }^{6}$ Our case 5 did not show any improvement on either the first day or one week post-operatively. Lack of improved vocal quality immediately following surgery may indicate a prolonged period of healing, rather than surgical failure, for a particular patient. Thus a longer period for evaluation of these patients is recommended to measure outcome success. ${ }^{6}$

Medialization thyroplasty using soft silicone implants has been shown to be safe and effective. ${ }^{7}$ Although the use of titanium implants have been shown to provide superior voice quality outcomes, the difference compared to soft silicone implants was insignificant. ${ }^{8}$ Complications ranging from implant extrusion to airway compromise are low as shown in our patients, reflecting other studies. ${ }^{9}$ Wound infection in one of our patients may have been caused by poor postoperative self-care. Implant extrusion is commonly caused by migration of the implant due to forceful cough ${ }^{9}$ but this possibility was minimized by the implant design used in the study. The implant was designed to have an anterior extension from the thyroid cartilage that holds and locks it in place. In addition, further modification of the implant allowed rotational adjustment without having to remove it from its insertion inside the thyroid cartilage, thus decreasing patient discomfort during surgery.

The approach to unilateral vocal cord paralysis is multidisciplinary, entailing a good clinical and voice history. For institutions without stateof-the-art equipment to assess voice quality, perceptual analysis of voice through the use of standardized surveys may provide substantial data to prognosticate treatment outcomes.

For glottal insufficiency, perceptual voice evaluation using selfadministered GFI and speech pathologist GRBAS assessment are important parameters in determining quality of life among patients with glottal insufficiency undergoing medialization laryngoplasty.

\section{REFERENCES}

1. Pavithran J, Menon JR. Unilateral vocal cord palsy: An etiopathological study. Int J Phonosurg Laryngol, 2011 Jan-Jun; 1(1):5-10.

2. Bach KK, Belafsky PC, Wasylik K, Postma GN, Koufman JA. Validity and reliability of the glottal function index. Arch Otolaryngol Head Neck Surg. 2005 Nov; 131(11):961-4.

3. Hirano M. Psycho-acoustic evaluation of voice. In: Arnold, Winckel, Wyke, eds. Disorders of Human Communication, 5, Clinical Examination of Voice. New York and Wien: Springer-Verlag; 1981:81-84.

4. Lore JM, Medina J. An Atlas of Head and Neck Surgery. $4^{\text {th }}$ edition. Philadelphia: Saunders; 2005.

5. Bhuta T, Patrick L, Garnett JD. Perceptual evaluation of voice quality and its correlation with acoustic measurements. J Voice. 2004 Sep;18(3):299-304.

6. Gorham MM, Avidano MA, Crary MA, Cotter CS, Cassisi NJ. Laryngeal recovery following type I thyroplasty. Arch Otolaryngol Head Neck Surg. 1998 Jul; 124(7);739-42.

7. Chrobok V, Pellant A, Sram F, Fric M, Praisler J, Prymula R, Svec JG. Medialization thyroplasty with a customized silicone implant: clinical experience. Folia Phoniatr Logop. 2008;60(2):91-6.

8. van Ardenne N, Vanderwegen J, Van Nuffelen G, De Bodt M, Van de Heyning P. . Medialization thyroplasty: vocal outcome of silicone and titanium implant. Eur Arch Otorhinolaryngol. 2011 Jan; 268(1):101-7.

9. Kartha S, Young K, Mohan S. Complications of medialization laryngoplasty (thyroplasty type-I) Int J Phonosurg Laryngol. 2011 Jan-Jun; 1(1):1-3. 